\title{
The Unified Theory of Science
}

\author{
Amagh Nduka \\ Departments of Physics and Mathematics, Federal University of Technology, Owerri, Nigeria \\ Email: amaghnduka@yahoo.com.au
}

Received 16 May 2016; accepted 22 July 2016; published 25 July 2016

Copyright (C) 2016 by author and Scientific Research Publishing Inc.

This work is licensed under the Creative Commons Attribution International License (CC BY).

http://creativecommons.org/licenses/by/4.0/

(c) (i) Open Access

\section{Abstract \\ This short expository note establishes that the whole of science is derivable from a certain cosmic parameter called the index of the universe.}

\section{Keywords}

Dimensionality, Partition, Primitive, Reducibility, Representation

\section{Introduction}

By science, we mean the study of nature. This study began in the $6 \mathrm{C}$ BC with the accidental discovery of electricity and magnetism by Thales of Miletus, the father of Greek science, and has flourished over the centuries.

An essential element in the study of science is the so called scientific method, which consists of two components. The first is the study of science based on experimentation; and the second component is the study of science via the mathematization (theorization) of nature's processes. The two components are not independent; a symbiotic relationship exists between them. A fundamental theory changes our view of the universe. Experiments confirm the predictions of the new theory. On the other hand, experiments may form the basis of a new theory, and the theory extends and generalizes the results of the experiment.

Between the $17 \mathrm{C}$ and early $20 \mathrm{C}$, mathematization of nature's processes was intellect-driven. The stupendous theories of Isaac Newton (classical mechanics and gravitation), J.C. Maxwell (electricity and magnetism), Albert Einstein (special relativity and electromagnetism), and P.A.M. Dirac (electron waves) were created at the time. All these theories, called the Classical Doctrine, were confirmed by experiments. The dividends of these spectacular theoretical achievements were the first and second industrial revolutions.

There was also a revolutionary development during the period - Dirac showed in 1928 that the electron states of the hydrogen atom were discrete, and that the electron had a twin called antielectron or positron. This amazing theoretical achievement was confirmed by experiments. The conclusion to be drawn from this is that a new kind of mathematics, distinct from those used in the construction of the Classical Doctrine, is required for the formulation of the theories of microphysical processes (atoms, molecules, nuclei). 
Completely oblivious of the Dirac data on the hydrogen electron and the fact that the Classical Doctrine was impotent in the explanation of experimental results on atomic processes around the end of the 19th and beginning of the 20th centuries theorists continued to apply classical mathematics. It then became necessary to adopt machine-driven approach as a replacement of the rather successful intellect-driven approach; and formal theories became a victim and were replaced by model theories. This approach created quantum electrodynamics (QED), electroweak theory (QFD), quantum chromodynamics (QCD), and the standard model. These model theories are at best incomplete theories which have introduced a plethora of unanswered questions. The disciples of the machine-driven method have continued to use their overwhelming political and economic clout to deny the world access to the truth. One obvious consequence is global warming which they erroneously ascribe to greenhouse gases, e.g. carbon dioxide.

\section{The Unified Theory of Science}

The starting point is the Dirac theory of the hydrogen atom electron. We invented the correct mathematics, called quantum mathematics (or discrete geometry), which generalizes the Dirac result to any atom. It turns out that all of science is derivable from a single cosmic parameter. We give hereunder a brief discussion of the work.

A set $\mathrm{S}$, whose elements are subsequently denoted by the symbol $|\mathrm{n}\rangle$ referred to as kets, is called an absolute space (or universe) if its elements do not obey the usual rules of a linear space. Here $n$, called the index of $\mathrm{S}$, takes on only non-negative integral and half-integral values excluding zero. The ket $|\mathrm{n}\rangle$ is concrete and absolute, $|\mathrm{n}\rangle=2\left(4^{\mathrm{n}}\right)$. Obviously these kets have nothing in common with the kets of elementary quantum mechanics.

A subspace (or world) $\mathrm{S}^{\prime}$ of $\mathrm{S}$ is defined by

1. Index $n$

2. Partition of $|n\rangle$ - by partition we mean the subdivision of $|n\rangle$ into its primitive geometrical entities.

3. The geometry of the entities is Euclidean (or pseudoeuclidean), classical (or discrete).

It is indeed amazing that the whole of science is derivable from the above scheme.

\section{Representation Theory}

By science we mean the representatives of the ket $|n\rangle$, called states, subject to the constraint that all states are electrically neutral.

\subsection{Classical Theories}

$\mathrm{n}=1,|\mathrm{n}\rangle=2(4)$. There are two independent partitions in this subspace, called our world:

$$
\begin{aligned}
& |1\rangle=(3,1 ; 3,1), \quad(3, \overline{3} ; 1,1) \\
& |1\rangle=(4 ; 4) .
\end{aligned}
$$

(1) gives the formal structures for classical particle theories of Newton; and (2) for classical wave theories of Einstein and Dirac [1]. We note in passing that J.C. Maxwell constructed in 1862 a theory of electricity and magnetism which is consistent with the first of (1). The structure of the Maxwell theory is wrong because it is based on secondary dynamical variables - to make the theory consistent with (2) an arbitrary constraint called gauge is imposed.

The structures (1) and (2) have a common origin, the ket $|1\rangle$, hence one can construct a unified theory of these theories, called Invariant Operator Theory [2].

\subsection{Quantum Theories}

We define the dimensionality of a state as a number equal to the algebraic sum of the primitive elements of a partition. This quantity is equal to eight for the Newton, Einstein, and Dirac theories. Because of wave-particle duality it must also apply to particulate states of the hydrogen atom, and must apply generally - this is dimensionality theorem. In this section we construct micro-physical states subject to the additional constraint imposed by the dimensionality theorem. 


\subsubsection{Fermion Theories}

$\mathrm{n}=1 / 2,1,2,3, \cdots, \infty$ in this subspace called the Fermion world.

$\mathrm{n}=1 / 2$ gives the Dirac solution with the partition.

$$
|1 / 2\rangle=2\left(4^{1 / 2}\right) \equiv(2, \overline{2})
$$

where $2=\overline{2}=(+,-),+=+1 / 2,-=-1 / 2$. Physically " 2 " and " $\overline{2}$ " stand for particle and antiparticle respectively.

But this solution is inconsistent with the dimensionality theorem, and must be replaced by

$$
|1 / 2\rangle \equiv(2, \overline{2} ; 2, \overline{2})
$$

the second entry of $(2, \overline{2})$ representing neutral spin $1 / 2$ particles (neutrinos and neutrons and their antiparticles). The Dirac solution has been generalized by us for the cases $n=1,2,3, \cdots, \infty$ [3].

The neutron is known to reside in the atomic nucleus. Until our work not much was known about the electron neutrino, but now the mystery surrounding this particle has disappeared [4].

The Fermion world consists of three subworlds, namely, the nucleus, lepton, and antilepton subworlds. The nucleus corresponding to $n=1 / 2$ is called alpha particle.

\subsubsection{Boson Theory}

$\mathrm{n}=1$ in this subspace called the boson world. The associated partitions are

$$
\begin{aligned}
& |1\rangle \equiv(3,1 ; 3,1) \\
& |1\rangle \equiv(1, \overline{3} ; 3, \overline{1})
\end{aligned}
$$

Partition (5) must be rejected because it is not independent of the classical partition (1). Thus, (6) gives the formal structure of the bosom state of rest.

The representation of (6) is a union of a spin zero particle, a spin zero antiparticle, a spin one particle, and a spin one antiparticle, corresponding to the photon, graviton, a vetor boson, and an antivector boson respectively. (6) then gives the unification of the fundamental interactions, with the vector boson a strongly or weakly interacting nuclear particle (strongly interacting if neutrons are involved, and weakly interacting if neutrinos are involved) [5].

The boson particle represented by (6) is electrically neutral. To ensure that the boson world and antiworld are electrically neutral nature provides muon and muon-neutrinos, and their antiparticles.

A survey of the literature of particle physics shows that Abdus Salam, Steven Weinberg, and Sheldon Glashow obtained a representation (electro-weak theory) consisting of five particles, namely, photon, three vector bosons, and Higgs boson. This was a laudable effort but the theory violates the cardinal constraints of electrical neutrality and dimensionality.

\subsubsection{Fermion-Boson Theories}

The final subspace $S$ ' which is independent of the foregoing subspaces is defined by $n=1,|1\rangle=2(4)$, and the partition,

$$
|1\rangle \equiv(2 ; 3, \overline{3}), \quad(3, \overline{3} ; \overline{2})
$$

Partition (7) gives the formal structure of a manifestly interacting world (chaotic world) - a world appropriate for astrophysics. The world is chaotic because it is impossible to construct $4 \times 4$ matrices from juxtaposition of a $2 \times 2$ and $6 \times 6$ matrices.

There are two representations which ensure electrical neutrality of the states. The first consists of a neutron or antineutron interacting with a charged vector boson and its antiparticle; and the second is interaction of a neutron or antineutron with a neutral vector boson and its antiparticle. In a low energy scenario, the neutron is replaced by a neutrino and the vector boson by a meson. The interaction involving neutrons and charged vector bosons is called normal star (or star), while the interaction involving neutrons and neutral vector bosons are called neutron star. These interactions, called strong nuclear interactions, occur in stars via a process called fusion, the telltale sign being light and particles from luminous stars like our sun [6]. Tau electrons, tau neutrinos and their antiparticles make the world of the normal star electrically neutral. 


\section{References}

[1] Nduka, A. (2012) The Geometrical Theory of Science. Applied Mathematics, 3, 1598-1600. http://dx.doi.org/10.4236/am.2012.311220

[2] Nduka, A. (2014) The Unified Geometrical Theory of Fields and Particles. Applied Mathematics. http://www.scirp.org/journal/AM/

[3] Nduka, A. (2014) The Geometrical Theory of the Structure of Nuclei, Atoms, and Molecules. Applied Mathematics. http://www.scrip.org/journal/AM/

[4] Nduka, A. (2013) The Neutrino Mass. Applied Mathematics, 4. http://www.scrip.org/journal/am/ http://dx.doi.org/10.4236/am.2013.42047

[5] Nduka, A. (2014) The Weak and Strong Nuclear Interactions. Applied Mathematics. http://www.scrip.org/journal/AM/

[6] Nduka, A. (2015) The World Energy Challenge and Global Warming. Energy and Power Engineering (EPE), 7. http://www.scrip.org/journal/EPE/

\section{Submit or recommend next manuscript to SCIRP and we will provide best service for you:}

Accepting pre-submission inquiries through Email, Facebook, LinkedIn, Twitter, etc.

A wide selection of journals (inclusive of 9 subjects, more than 200 journals)

Providing 24-hour high-quality service

User-friendly online submission system

Fair and swift peer-review system

Efficient typesetting and proofreading procedure

Display of the result of downloads and visits, as well as the number of cited articles

Maximum dissemination of your research work

Submit your manuscript at: http://papersubmission.scirp.org/ 